\title{
Estimativas de parâmetros genéticos para características de crescimento em bovinos da raça Canchim utilizando modelos de dimensão finita ${ }^{1}$
}

\author{
Fernando Baldi², Mauricio Mello de Alencar ${ }^{3}$, Lucia Galvão de Albuquerque ${ }^{2}$ \\ 1 Trabalho financiado pela FAPESP. \\ 2 Programa de Pós-graduação em Genética e Melhoramento Animal - UNESP, Campus de Jaboticabal, SP, CEP: $14884-900$. \\ ${ }^{3}$ Embrapa Pecuária Sudeste, CEP:13560-970, São Carlos, SP, Brasil.
}

RESUMO - Os objetivos neste trabalho foram avaliar diferentes modelos, em relação aos efeitos maternos considerados, para características de crescimento e estimar os parâmetros genéticos para essas características em bovinos da raça Canchim, por meio de análises uni, bi e multicaracterísticas. As características peso ao nascimento, peso ao desmame, pesos padronizados para 12, 18, 24 e 30 meses de idade em machos e fêmeas e peso adulto de fêmeas foram analisadas utilizando-se quatro modelos com os efeitos aleatórios adicionados em sequência. Os efeitos maternos influenciaram os pesos do nascimento aos 2 anos de idade e o peso à desmama foi o mais afetado pelos efeitos maternos. As estimativas de herdabilidade direta obtidas das análises bi e multicaracterísticas foram superiores àquelas obtidas das análises unicaracterísticas. As estimativas de herdabilidade do efeito genético direto obtidas usando análise multicaracterística foram 0,39 para peso ao nascer; 0,31 para peso à desmama; 0,29 para peso aos 12 meses; 0,28 para peso aos 18 meses; 0,26 para peso aos 24 meses; 0,30 para peso aos 30 meses; e 0,38 para peso à idade adulta. As correlações genéticas estimadas entre pesos obtidos em idades jovens com peso à idade adulta foram altas, acima de 0,79. A seleção com base em características de crescimento em qualquer idade pode promover ganhos genéticos moderados no peso corporal de animais da raça Canchim em todas as idades-padrão, inclusive nos pesos ao nascer e à idade adulta das fêmeas. É importante considerar nas análises os pesos prévios à seleção para estimar parâmetros genéticos para pesos após a seleção. A análise multicaracterística é a mais indicada.

Palavras-chave: análise multicaracterística, bovinos de corte, correlação genética, herdabilidade, peso padronizado

\section{Estimation of genetic parameters for growth traits in Canchim cattle with finite models}

\begin{abstract}
The objectives of this work were to evaluate different models, regarded to considered maternal effects, for growth traits and to estimate genetic parameters for these traits in Canchim breed bovine, by one, two and multitrait analyses. The weight traits at birth, at weaning, standardized weight for 12, 18, 24 and 30 months of age in males and females, and mature female weight were analyzed using four models with random effects added in sequence. Maternal effects influenced birth weight at 2 years of age and weaning weight was the most affected by maternal effects. Direct heritability estimates obtained by two- and multitrait analyses were higher than those obtained through one-trait analyses. Estimates of direct genetic effect heritability obtained using multi-trait analyses were 0.39 for weight at birth; 0.31 for weight at weaning; 0.29 for weight at 12 months of age; 0.28 for weight at 18 months of age; 0.26 for weight at 24 months of age; 0.30 for weight at 30 months of age and 0.38 for weight at mature age. Genetic correlations estimated among weights at early ages and mature weight were high, above 0.79 . Selection based on growth traits at any age can promote intermediate genetic gains on the body weight of Canchim breed animals at all standard ages, including weight at birth and at mature age of females. It is important to consider in the analyses the weights previous to selection to estimate genetic parameters for weights after selection. Multitrait analyses is the best indicated.
\end{abstract}

Key Words: beef cattle, genetic correlation, heritability, multitrait analyses, weight at standard age

\section{Introdução}

No Brasil, os programas de melhoramento genético para raças bovinas de corte têm priorizado a seleção para características de crescimento, como o peso ou ganho de peso em determinados períodos (Alencar, 2002). Uma das considerações-chave no momento de avaliar as consequências do maior crescimento é seu impacto sobre o rebanho de vacas, particularmente sobre os custos de manutenção. Aproximadamente $50 \%$ de toda energia requerida pelo rebanho é destinada ao atendimento das necessidades energéticas de manutenção das matrizes 
(Montaño-Bermudez et al., 1990). O requerimento energético para manutenção está diretamente ligado ao peso vivo e à condição corporal da vaca (Klosterman et al., 1968; Owens et al., 1993). A seleção para adequar o peso ou o tamanho à idade adulta da vaca às condições de alimentação e manejo enquanto se aumenta o peso à desmama e ao abate da progênie requer estimativas acuradas de parâmetros genéticos de pesos em diferentes idades.

Em gado de corte, os efeitos maternos são importantes para as características de crescimento até a desmama, embora efeitos significativos permaneçam após a desmama e tenham sido identificados para pesos em idades avançadas (Meyer et al., 1993; Rumph et al., 2002; Cyrillo et al., 2004). Segundo Meyer (1997), os modelos que não incluem os efeitos maternos conduzem a superestimativas de herdabilidade direta. Muitas vezes os efeitos maternos não são considerados na estimação de parâmetros genéticos em gado de corte, em razão da necessidade de uma estrutura de dados adequada para sua obtenção e também por incrementarem a complexidade das análises, sobretudo as análises bi e multicaracterísticas.

Para bovinos de corte, Meyer (1995) e Kaps et al. (1999) afirmaram que uma forma de considerar os efeitos da seleção sobre a variância genética é utilizar um modelo multicaracterístico que inclua os pesos prévios à seleção e, desta forma, corrigir o viés na variância genética. Na literatura são escassos os trabalhos em gado de corte (Meyer et al., 1993; Meyer, 1995; Kaps et al., 1999; Mercadante et al., 2004; Boligon et al., 2009) que tenham utilizado modelo multicaracterística visando estimar parâmetros genéticos para características de crescimento, sendo que, na maioria dos trabalhos, foram utilizadas análises bicaracterísticas.

Os objetivos neste estudo foram avaliar diferentes modelos de seleção com base nos efeitos aleatórios maternos considerados para características de crescimento e estimar parâmetros genéticos para pesos do nascimento à idade adulta (pesos ao nascer, à desmama e aos 12, 18, 24 e 30 meses de idade e o peso à idade adulta somente nas fêmeas) para bovinos da raça Canchim, em análises uni, bi e multicaracterísticas.

\section{Material e Métodos}

Os dados analisados são provenientes do rebanho da raça Canchim da Embrapa Pecuária Sudeste, localizada no município de São Carlos, São Paulo. Os animais nasceram entre os anos de 1954 e 2005 e foram criados exclusivamente em pastagens de diferentes gramíneas com suplementação mineral durante todo o ano.
Até 1978, as pesagens de todos os animais do rebanho eram realizadas mensalmente nos dois primeiros dias úteis de cada mês. A partir de 1979, os animais passaram a ser pesados trimestralmente (em janeiro, abril, julho e outubro) e as vacas passaram a ser pesadas após o parto, ao desmame dos bezerros, à entrada e saída da estação de monta e na inseminação. A partir de 1980, as pesagens trimestrais deixaram de ser feitas e os animais passaram a ser pesados ao nascimento, ao desmame e aos 12, 18, 24 e 30 meses de idade. Maiores informações sobre as condições de alimentação e manejo dos animais podem ser obtidas em Mello et al. (2002).

A seleção de machos e fêmeas para reprodução foi realizada em três etapas: na época da desmama (8 a 9 meses de idade); ao completar um ano ou um ano e meio de idade; e aos dois anos ou dois anos e meio de idade. A escolha dos touros foi baseada em características de crescimento (peso por idade), conformação, estética, precocidade, temperamento, pelagem, tipo e fertilidade (Barbosa, 1997). A partir de 1979, a seleção dos touros dentro de cada ano, foi feita com base em características raciais e peso padronizado para 365 dias de idade, ajustado para mês de nascimento e idade da mãe ao parto. Além disto, os animais foram submetidos a exame andrológico com cerca de 24 meses de idade. A seleção de machos jovens, desde 1996, passou a ser feita pela diferença esperada na progênie (DEP) direta para peso aos 12 meses de idade. A mensuração do perímetro escrotal aos 12, 18 e 24 meses de idade iniciou-se em 1982 e também foi considerada como critério de seleção. A partir de 2000, a seleção dos machos passou a ser feita com base em características raciais e nas diferenças esperadas na progênie para peso e perímetro escrotal ao sobreano.

Até o ano de 1975, as novilhas entravam em reprodução com aproximadamente 34 meses de idade e $360 \mathrm{~kg}$ de peso vivo, mas, a partir de 1976, esses critérios foram mudados para 24 a 28 meses e cerca de $300 \mathrm{~kg}$ de peso vivo. Em geral, as vacas eram colocadas com touros após a desmama dos bezerros, ou seja, 7 a 8 meses após a parição. Esse tipo de manejo foi modificado em 1969, quando todas as vacas que pariram antes do início da estação de monta foram colocadas em reprodução. Nova mudança ocorreu a partir de 1976 (exceto 1977, quando o manejo anterior foi utilizado), quando todas as vacas foram colocadas com touros durante a estação de monta, com exceção daquelas prenhes da estação anterior e que iriam parir após o término da estação vigente. Durante as estações de monta, cada touro era colocado com aproximadamente 30 vacas, em piquetes isolados. A partir de 1979, começou-se a utilizar também a inseminação 
artificial. Em vários anos, foram utilizadas duas estações de monta, uma no primeiro semestre e outra no segundo, mas que não tinham mês fixo para iniciar nem para terminar, e cuja duração também variava (2 a 4 meses). A eliminação de vacas do rebanho se deu principalmente por motivos de doença e/ou acidente; contudo, a partir de 1977, iniciou-se o descarte de vacas consideradas de fertilidade mais baixa, ou seja, que saíssem vazias de duas estações de monta consecutivas. A partir de 1979, a seleção de novilhas para entrada em reprodução foi feita antes do início da estação de monta, por volta dos 22 meses de idade, considerando, além das características raciais, o desenvolvimento dos animais. Procurou-se manter no rebanho apenas novilhas prenhes da primeira estação de monta.

Neste trabalho foram estudadas as características peso ao nascimento, peso à desmama padronizado para 240 dias de idade e pesos padronizados para 12, 18, 24 e 30 meses de idade para machos e fêmeas e, somente no caso das fêmeas, o peso à idade adulta, que foi considerado como o peso logo após o parto, para vacas de 4, 5, 6 a 8 e acima de 9 anos de idade. Foi considerado apenas um peso para cada vaca adulta e, no caso de a vaca possuir mais de um peso, utilizou-se o peso mais próximo de 6 anos, idade em que o animal provavelmente já parou de crescer (Silva et al., 2000).

As variâncias e covariâncias foram estimadas pelo método da máxima verossimilhança restrita (REML) em análises uni, bi e multicaracterísticas. Para as análises uni e bicaracterísticas, foi utilizado o software MTDFREML (Boldman et al., 2002) e, para a análise multicaracterística, o programa REMLF90 (Misztal, 2001), que utiliza o algoritmo de maximização da esperança, com processo de aceleração da convergência. As médias das variâncias genéticas e fenotípicas para cada característica obtidas nas análises bicaracterísticas foram usadas para estimação da herdabilidade, que, posteriormente, foi comparada à estimativa de herdabilidade obtida pela análise unicaracterística e multicaracterística. Nas análises bicaracterísticas, foram feitas todas as combinações entre as características. Admitiu-se que a convergência era atingida quando o quadrado das diferenças relativas entre estimativas consecutivas era menor que $10^{-9}$.
No modelo de análise, considerou-se efeito fixo o grupo de contemporâneos, a idade da mãe ao parto como covariável (efeito linear e quadrático) para os pesos ao nascer, à desmama, aos 12, 18 e aos 24 meses de idade, os efeitos linear da idade do animal à pesagem (com exceção do peso ao nascer) e os efeitos linear e quadrático da idade da fêmea ao parto para o peso à idade adulta das fêmeas. O grupo de contemporâneos foi definido como animais do mesmo sexo, mês e ano de nascimento para os pesos ao nascer, à desmama, aos 12, 18, 24 e 30 meses de idade. Para peso à idade adulta, o grupo de contemporâneos foi definido para animais que nasceram no mesmo ano e mês. Grupos de contemporâneos com menos de três observações foram excluídos. Foram excluídos registros de pesos fora dos intervalos dados pela média do grupo de contemporâneos mais ou menos três desvios-padrão (Tabela 1).

Primeiramente, foram realizadas análises unicaracterísticas para todos os pesos estudados aplicando-se modelos que diferiram quanto aos efeitos aleatórios: modelo 1 (M1): efeito genético direto; modelo 2 (M2): efeito genético direto + efeito de ambiente permanente materno; modelo 3 (M3): efeito genético direto + efeito genético materno; modelo 4 (M4): efeito genético direto + efeito genético materno + efeito de ambiente permanente materno. Para verificar a significância dos efeitos aleatórios incluídos no modelo e definir os modelos mais adequados para as análises bicaracterísticas e a multicaracterística, foi utilizado o teste de razão de verossimilhança (LRT), que verifica a diminuição do valor de -2 Log L pela inclusão de mais um parâmetro no modelo, o qual segue distribuição de Qui-quadrado com $g$ graus de liberdade, em que $g$ é a diferença em números de parâmetros estimados entre os dois modelos comparados (Dobson, 1990). Neste teste, a probabilidade de erro utilizada foi de $1 \%$. O modelo geral utilizado foi:

$$
\mathrm{y}=\mathrm{Xb}+\mathrm{Z}_{1} \mathrm{a}+\mathrm{Z}_{2} \mathrm{~m}+\mathrm{Z}_{3} \mathrm{C}+\mathrm{e}
$$

em que $\mathrm{y}=$ vetor das características observadas; $\mathrm{b}=$ vetor de efeitos fixos; a = vetor de efeitos genéticos aditivos diretos; $\mathrm{m}$ = vetor de efeitos genéticos aditivos maternos; $\mathrm{c}=$ vetor de efeitos de ambiente permanente materno; $\mathrm{e}=$ vetor de efeitos residuais, $\mathrm{X}, \mathrm{Z}_{1}, \mathrm{Z}_{2}$ e $\mathrm{Z}_{3}=$ matrizes de incidência relacionando b, a, m e c a y. Neste estudo,

Tabela 1 - Estrutura geral do arquivo e descrição dos dados para pesos em várias idades

\begin{tabular}{|c|c|c|c|c|c|c|c|c|c|}
\hline Característica & $\mathrm{N}^{\circ}$ observações & Touros & Vacas & Grupo de & contemporâneos & Média & Desvio-padrão & Mínimo & Máximo \\
\hline Peso ao nascer & 8.360 & 321 & 2.440 & & 531 & 35,1 & 5,4 & 15 & 65 \\
\hline Peso à desmama & 7.531 & 320 & 2.325 & & 511 & 203,5 & 35,3 & 90 & 300 \\
\hline Peso aos 12 meses & 6.515 & 317 & 2.224 & & 475 & 219,9 & 44,6 & 100 & 400 \\
\hline Peso aos 24 meses & 3.760 & 250 & 1.535 & & 361 & 336,5 & 58,1 & 180 & 501 \\
\hline Peso aos 30 meses & 2.060 & 185 & 1.099 & & 224 & 394,8 & 66,2 & 220 & 580 \\
\hline Peso à idade adulta & 1.098 & 189 & 746 & & 144 & 499,4 & 66,3 & 320 & 734 \\
\hline
\end{tabular}


assumiu-se que $\mathrm{E}[\mathrm{y}]=\mathrm{Xb} ; \operatorname{Var}(\mathrm{a})=\mathrm{A} \otimes \mathrm{S}_{\mathrm{a}} ; \operatorname{Var}(\mathrm{m})=\mathrm{A} \otimes \mathrm{S}_{\mathrm{m}}$; $\operatorname{Var}(\mathrm{c})=\mathrm{I} \otimes \mathrm{S}_{\mathrm{c}}$ e $\operatorname{Var}(\mathrm{e})=\mathrm{I} \otimes \mathrm{S}_{\mathrm{e}}$, em que $\mathrm{S}_{\mathrm{a}}=$ matriz de covariâncias genéticas aditivas; $\mathrm{S}_{\mathrm{m}}=$ matriz de covariâncias genéticas maternas; $\mathrm{S}_{\mathrm{c}}=$ matriz de covariâncias de ambiente permanente materno; $\mathrm{S}_{\mathrm{e}}=$ matriz de covariância residual; A = matriz do numerador de relações genético-aditivas; $\mathrm{I}=$ matriz identidade; $\mathrm{e} \otimes=$ produto direto entre matrizes. Assumiu-se ainda que os vetores a, m e c não se correlacionam entre si. No caso da análise unicaracterística, o erro-padrão das estimativas de herdabilidade foi obtido utilizando a seguinte expressão: erro-padrão $=s / \sqrt{N}$, em que $S$ = desvio-padrão das estimativas de herdabilidade; $N=$ número de observações.

Em todas as análises, foi utilizado um arquivo de pedigree contendo identificação do animal, do pai e da mãe, totalizando 12.485 animais, 385 touros e 2.946 mães na matriz de parentesco. Os dados analisados incluíram, em média, vacas com 3,68 filhos, touros com 13,6 filhas e 59,3 netos(as).

\section{Resultados e Discussão}

Com exceção do peso aos 30 meses e do peso à idade adulta, houve redução significativa do -2 Log L estimado quando os efeitos genético materno e de ambiente permanente materno foram incluídos no modelo de análise, em relação ao modelo que considerava como efeito aleatório apenas o efeito genético aditivo direto dos animais (Tabela 2).

Os resultados indicaram que os efeitos maternos afetam o peso, inclusive após o desmame, provavelmente pelo fato de que efeitos residuais permanecem após essa idade, afetando, portanto, o crescimento dos animais. O modelo mais adequado para estimar parâmetros genéticos para os pesos ao nascer, à desmama e aos 12, 18 e 24 meses de idade seria o completo, aquele que incluísse os efeitos aleatórios genético direto e materno e de ambiente permanente materno e, para peso aos 30 meses de idade e peso à idade adulta seria o modelo que incluísse somente o efeito genético direto. Rumph et al. (2002), analisando dados de vacas da raça Hereford, observaram que o modelo mais adequado para estimar parâmetros genéticos para peso à idade adulta foi aquele que incluiu os efeitos genéticos direto e materno. Em bovinos da raça Hereford e Wokalup, Meyer et al. (1993) notaram que os efeitos maternos influenciaram os pesos do nascimento até os 600 dias de idade. De acordo com Cyrillo et al. (2004), em bovinos da raça Nelore, os efeitos maternos influenciaram o crescimento do nascimento até os 378 dias de idade. Segundo Meyer (1997), os modelos que não incluíram os efeitos maternos conduzem a superestimativas da herdabilidade direta. Portanto, visando à obtenção de parâmetros genéticos mais acurados para pesos após o desmame, seria recomendável considerar os efeitos maternos no modelo de avaliação.

Com exceção do peso aos 24 meses de idade, as estimativas de variância genética aditiva nas análises bi e multicaracterísticas foram superiores às estimadas em análise unicaracterística para os pesos a partir de 1 ano de idade (Figura 1). De acordo com Bulmer (1971), Meyer \& Thompson (1984) e Henderson (1986), as variâncias genéticas da população podem mudar com a seleção. Segundo Meyer (1995), o aumento da variância genética aditiva nas análises multicaracterísticas reflete a remoção do vício provocado pela seleção. Mercadante et al. (2004) e Boligon et al. (2009) obtiveram resultados semelhantes aos obtidos neste trabalho quando analisaram os pesos à seleção em conjunto com o peso à idade adulta em análises bi e multicaracterísticas. Do mesmo modo, avaliando pesos do nascimento até os 600 dias de idade, Meyer et al. (1993) observaram aumento nas estimativas da variância genética aditiva em análises envolvendo três ou quatro pesos simultaneamente, em relação à análise unicaracterística.

Neste estudo, a seleção dos animais para reprodução foi praticada com base nos pesos ao desmame e ao ano de idade, portanto, a utilização dos pesos obtidos antes e

Tabela 2 - Mudanças nas estimativas do -2 Log L e sua significância causada pelo uso de diferentes modelos (M) para a análise dos pesos em várias idades

\begin{tabular}{|c|c|c|c|c|}
\hline \multirow[b]{2}{*}{ Característica } & \multicolumn{4}{|c|}{ Mudança do -2 $\log \mathrm{L}^{1}$ estimado } \\
\hline & $\mathrm{M} 1-\mathrm{M} 2^{1}$ & M1 - M3 & M1 - M4 & M2 - M4 \\
\hline Peso ao nascer & $64 * *$ & $79 * *$ & $88 * *$ & $24 * *$ \\
\hline Peso à desmama & $283 * *$ & $257 * *$ & $307 * *$ & $24 * *$ \\
\hline Peso aos 12 meses & $67 * *$ & $61 * *$ & $77 * *$ & $10 * *$ \\
\hline Peso aos 18 meses & $29 * *$ & $46 * *$ & $47 * *$ & $18 * *$ \\
\hline Peso aos 24 meses & $7 * *$ & $14 * *$ & $14 * *$ & $7 * *$ \\
\hline Peso aos 30 meses & $0,28 \mathrm{NS}$ & $3,24 \mathrm{NS}$ & & \\
\hline Peso à idade adulta das fêmeas & $0,01 \mathrm{NS}$ & $0,06 \mathrm{NS}$ & & \\
\hline
\end{tabular}

${ }^{1}$ M1: efeito genético direto; M2: efeito genético direto + efeito de ambiente permanente materno; M3: efeito genético direto + efeito genético materno; M4: efeito genético direto + efeito genético materno+efeito de ambiente permanente materno; **P<0,01, NS: não-significativo. -2 Log L = função de verossimilhança. 

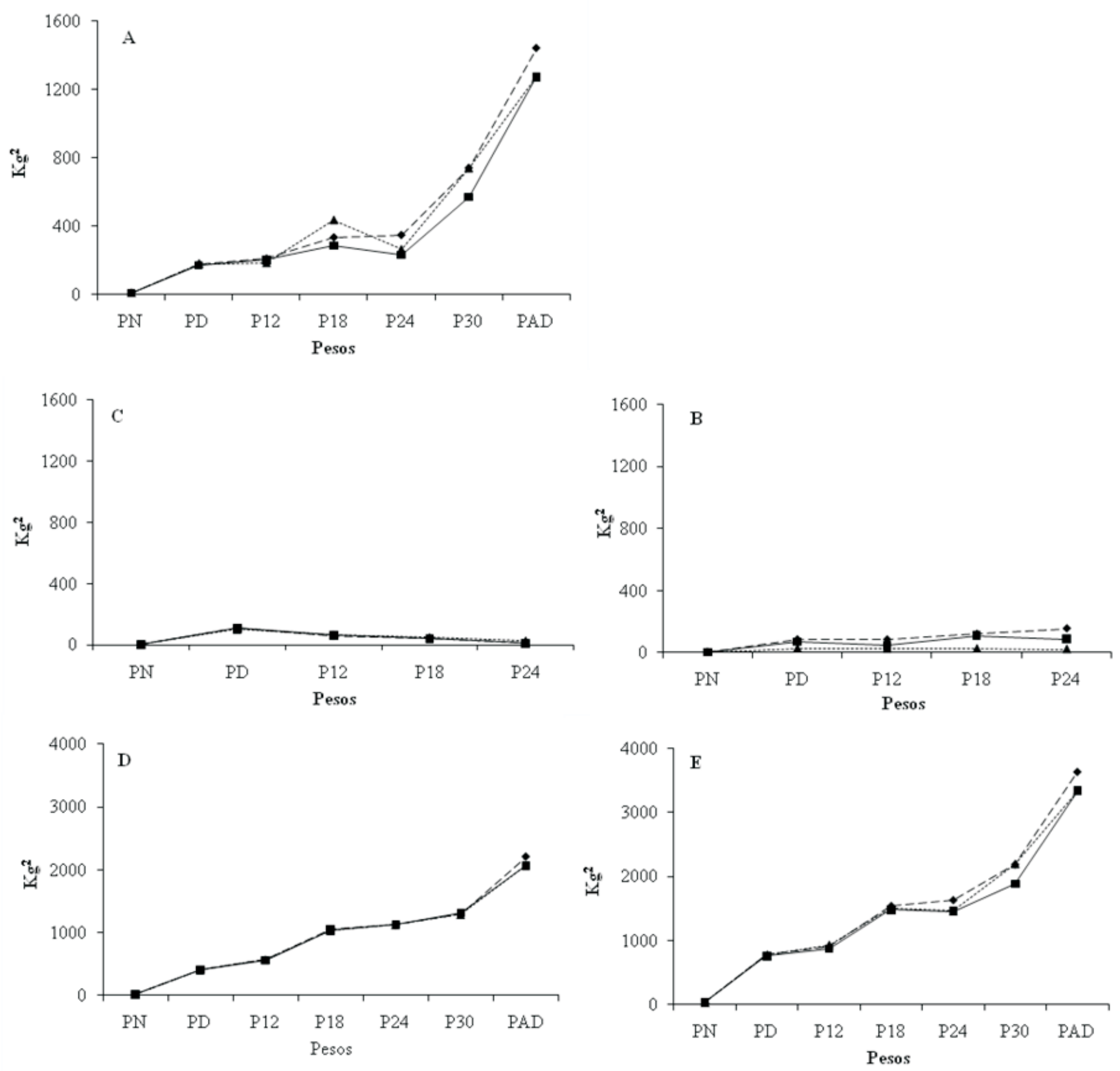

\footnotetext{
(-) análises unicaracterística

(•) análises bicaracterística

( $\boldsymbol{\Delta}$ ) análise multicaracterística
}

Figura 1 - Variâncias genética aditiva (A), genética materna (B), de ambiente permanente materno (C), residual (D) e fenotípica (E) para os pesos ao nascer (PN), à desmama (PD), aos 12 (P12), 18 (P18), 24 (P24) e 30 (P30) meses de idade e à idade adulta (PAD).

após a seleção em análises bi e multicaracterísticas permitiu remover da variância genética aditiva o viés devido à seleção. De acordo com Meyer (1995) e Kaps et al.(1999), em rebanhos com seleção a idades jovens, os pesos tomados nestas idades poderiam ser analisados juntamente com o peso à idade adulta para incluir o viés causado pela seleção.

Nas análises bi e multicaracterísticas a partir dos 18 meses de idade, a variância fenotípica foi superior àquela obtida na análise unicaracterística, provavelmente em decorrência do aumento da variância genética aditiva partir dessa idade (Figura 1). No caso das variâncias residuais, não houve diferenças na magnitude das variâncias obtidas das análises uni, bi e multicaracterísticas. No entanto, Boligon et al. (2009) encontraram menores variâncias fenotípicas e residuais em análises bi e multicaracterísticas para pesos a partir do sobreano. 
A variância do ambiente permanente materno apresentou valor máximo à desmama, e não houve diferenças nas estimativas obtidas das análises uni, bi e multicaracterísticas. Após a desmama, a variância do ambiente materno diminuiu gradualmente até os 24 meses de idade. O mesmo comportamento observado neste estudo foi relatado por Meyer et al. (1993), Albuquerque \& Meyer (2001), Mucari \& Oliveira (2003), Dias et al. (2005) e Boligon et al. (2009) para a variância do ambiente permanente materno. No caso da variância genética materna, as estimativas obtidas das análises uni e bicaracterísticas foram superiores àquelas obtidas a partir da análise multicaracterística. A menor magnitude da variância genética materna obtida na análise multicaracterística provavelmente é consequência de diferenças na partição das variâncias genética direta e materna em relação às análises uni e bicaracterísticas.

Em termos gerais, as estimativas de herdabilidade direta pelas análises bi e multicaracterísticas foram maiores que as obtidas pelas análises unicaracterísticas, principalmente para pesos após o sobreano (Tabela 3). As estimativas obtidas pelas análises bicaracterísticas foram superiores àquelas determinadas pelas análises unicaracterísticas a partir dos 12 meses de idade e até a idade adulta, enquanto aquelas obtidas pela análise multicaracterística foram superiores às encontradas pelas análises unicaracterísticas a partir do nascimento e até os 30 meses de idade. Em bovinos da raça Nelore, Boligon et al. (2009) obtiveram estimativas de herdabilidade diretas superiores para pesos após a seleção em análises bi e multicaracterísticas em relação às obtidas por análises unicaracterísticas. Meyer et al. (1993) obtiveram maiores estimativas de herdabilidade direta para pesos após a seleção quando pesos prévios à seleção foram considerados em análise multicaracterística. Kaps et al. (1999), em bovinos da raça Angus, obtiveram aumento na herdabilidade para peso assintótico adulto quando o peso à desmama foi considerado na análise. Mercadante et al. (2004), utilizando pesos de fêmeas da raça Nelore, relataram estimativas de herdabilidade para peso ao início da monta superiores quando os pesos à seleção foram incluídos em análises trivariadas. Portanto, seria recomendável na hora de estimar a herdabilidade para pesos após a seleção, incluir os pesos anteriores à seleção de forma a incrementar as estimativas de herdabilidade e diminuir o viés causado pela seleção.

As estimativas de herdabilidades diretas para peso ao nascer foram semelhantes às relatadas por Freitas et al. (1994), Alencar et al. (1998) e Mello et al. (2002), de 0,33; 0,39 e 0,39,

Tabela 3 -Estimativas de parâmetros genéticos e ambientais para pesos em várias idades obtidas por análises uni, bi e multicaracterísticas

\begin{tabular}{|c|c|c|c|c|}
\hline Características & $h_{d}^{2}$ & $h_{m}^{2}$ & $c^{2}$ & $e^{2}$ \\
\hline & \multicolumn{4}{|c|}{ Análise unicaracterística } \\
\hline Peso ao nascer & $0,34 \pm 0,03$ & $0,06 \pm 0,02$ & $0,04 \pm 0,01$ & $0,56 \pm 0,03$ \\
\hline Peso à desmama & $0,23 \pm 0,03$ & $0,09 \pm 0,02$ & $0,14 \pm 0,02$ & $0,54 \pm 0,03$ \\
\hline Peso aos 12 meses & $0,23 \pm 0,03$ & $0,05 \pm 0,02$ & $0,07 \pm 0,02$ & $0,64 \pm 0,03$ \\
\hline Peso aos 18 meses & $0,19 \pm 0,03$ & $0,07 \pm 0,02$ & $0,03 \pm 0,02$ & $0,71 \pm 0,03$ \\
\hline Peso aos 24 meses & $0,16 \pm 0,04$ & $0,06 \pm 0,03$ & $0,01 \pm 0,02$ & $0,78 \pm 0,03$ \\
\hline Peso aos 30 meses & $0,30 \pm 0,05$ & & & $0,70 \pm 0,05$ \\
\hline \multirow[t]{2}{*}{ Peso à idade adulta } & $0,38 \pm 0,08$ & & & $0,62 \pm 0,08$ \\
\hline & \multicolumn{4}{|c|}{ Análise bicaracterística } \\
\hline Peso ao nascer & 0,35 & 0,06 & 0,04 & 0,55 \\
\hline Peso à desmama & 0,23 & 0,11 & 0,14 & 0,52 \\
\hline Peso aos 12 meses & 0,23 & 0,09 & 0,07 & 0,61 \\
\hline Peso aos 18 meses & 0,22 & 0,08 & 0,03 & 0,67 \\
\hline Peso aos 24 meses & 0,21 & 0,09 & 0,01 & 0,69 \\
\hline Peso aos 30 meses & 0,34 & & & 0,66 \\
\hline \multirow[t]{2}{*}{ Peso à idade adulta } & 0,39 & & & 0,61 \\
\hline & \multicolumn{4}{|c|}{ Análise multicaracterística } \\
\hline Peso ao nascer & 0,39 & 0,03 & 0,05 & 0,53 \\
\hline Peso à desmama & 0,31 & 0,03 & 0,13 & 0,53 \\
\hline Peso aos 12 meses & 0,29 & 0,02 & 0,07 & 0,62 \\
\hline Peso aos 18 meses & 0,28 & 0,01 & 0,03 & 0,68 \\
\hline Peso aos 24 meses & 0,26 & 0,01 & 0,02 & 0,71 \\
\hline Peso aos 30 meses & 0,30 & & & 0,70 \\
\hline Peso à idade adulta & 0,38 & & & 0,62 \\
\hline
\end{tabular}

$h_{d}^{2}$ : herdabilidade direta; $h_{m}^{2}$ : herdabilidade materna; $c^{2}$ : proporção da variância de ambiente permanente materno em relação à variância fenotípica total; $e^{2}$ erro. 
respectivamente, para um conjunto de dados do mesmo rebanho utilizado neste trabalho. Em bovinos da raça Canchim, Mello et al. (2002) e Mello et al. (2006) obtiveram estimativas de herdabilidade superiores para pesos à desmama e aos 12 meses de idade, variando de 0,38 a 0,63, empregando os métodos da máxima verossimilhança restrita e inferência bayesiana, respectivamente. Boligon et al. (2009), utilizando bovinos da raça Nelore, obtiveram estimativas de herdabilidade para pesos aos 18 e aos 24 meses de idade superiores em comparação às obtidas neste trabalho. Utilizando fêmeas da raça Canchim, Baldi et al. (2006) relataram estimativa de herdabilidade semelhante $(0,23)$ às obtidas neste trabalho para pesos à entrada da primeira monta, próximo aos 24 meses de idade.

As estimativas de herdabilidade direta para peso à idade adulta obtidas neste estudo foram inferiores àquelas encontradas por Mello et al. (2006), de 0,54, e Baldi et al. (2006), 0,46, utilizando bovinos da raça Canchim. Rosa et al. (2000, 2001), Silva et al. (2000), Talhari et al. (2003) e Mercadante et al. (2004), em análises unicaracterísticas, obtiveram valores de herdabilidade que variaram entre 0,25 e 0,52. Em trabalhos (Meyer, 1995; Johnston et al., 1996; Arango et al., 2002; Choy et al., 2002; Mercadante et al., 2004; Nephawe, 2004) com medidas repetidas ou análises multicaracterísticas do peso, os valores de herdabilidade variaram de 0,28 a 0,54 . Quando o peso adulto foi estimado por meio da modelagem de curvas de crescimento, Johnson et al.(1990), Meyer(1995), Kaps et al.(1999), Silva et al. (2000) e Talhari et al. (2003), os valores de herdabilidade variaram de 0,38 a 0,73. Lôbo et al. (2000) relataram herdabilidade de 0,28 para peso adulto da vaca como média de cinco estudos com bovinos criados em países tropicais, enquanto Koots et al. (1994) observaram valor bem superior $(0,50)$ para a mesma característica, média de 25 estudos com bovinos criados em sua maioria em países de clima temperado.

No caso do efeito materno, as estimativas de herdabilidade foram de magnitudes baixas e semelhantes entre as análises uni e bicaracterísticas, e superiores àquelas obtidas na análise multicaracterística, na qual os menores valores de herdabilidade materna estiveram associados a maiores valores de herdabilidade direta, possivelmente em razão de diferenças na partição das variâncias genéticas direta e materna para pesos do nascimento até os 24 meses de idade. Para pesos após desmama, Meyer et al. (1993) utilizaram análise multicaracterística e obtiveram herdabilidades materna superiores e estimativas do efeito de ambiente permanente materno inferiores em relação àquelas obtidas na análise univariada. As estimativas de herdabilidade materna em análises uni e bicaracterísticas foram semelhantes às relatadas por Alencar et al. (1998), com valores de 0,04 para peso ao nascer, 0,10 para peso à desmama e 0,03 para peso aos 12 meses. No entanto, Mello et al. (2002) obtiveram estimativas inferiores para peso ao nascer $(0,03)$ e à desmam $(0,04)$ e semelhantes para peso aos 12 meses de idade $(0,05)$.

As estimativas do efeito de ambiente permanente materno obtidas neste estudo foram semelhantes entre as análise uni, bi e multicaracterísticas. Mello et al. (2002), para os pesos ao nascer, à desmama e aos 12 meses, obtiveram valores semelhantes aos deste estudo, com valores de 0,06 ; 0,14 e 0,09 , respectivamente. Neste trabalho, as estimativas da variância genética materna obtidas pela análise multicaracterísticas, uni e bicaracterísticas diferiram. Isto mostra uma partição de variância diferente para cada modelo. Meyer et al. (1993) relataram diferente partição das variâncias genética materna e de ambiente permanente materno para pesos pós-desmama em análises uni, bi e multivariadas.

As correlações genéticas entre as características foram de magnitude média a alta e as correlações obtidas da análise multicaracterística foram, em termos gerais, superiores àquelas obtidas das análises bicaracterísticas (Tabela 4). As correlações residuais obtidas por análises bicaracterística e multicaracterística foram, no entanto, de magnitude semelhante. Para pesos de bovinos da raça Hereford em diferentes idades, Meyer et al. (1993) relataram correlações genéticas semelhantes em análises bi e multicaracterísticas. Segundo esses autores, as correlações genéticas são menos afetadas pelo viés ou desvio causado

Tabela 4 - Estimativas das correlações genéticas (acima da diagonal) e residuais (abaixo da diagonal), entre as características de crescimento, obtidas em análise bi e multicaracterística (entre parênteses)

\begin{tabular}{lccccccc}
\hline Característica $^{1}$ & $\begin{array}{c}\text { Peso ao } \\
\text { nascer }\end{array}$ & $\begin{array}{c}\text { Peso à } \\
\text { desmama }\end{array}$ & $\begin{array}{c}\text { Peso aos } \\
12 \text { meses }\end{array}$ & $\begin{array}{c}\text { Peso aos } \\
18 \text { meses }\end{array}$ & $\begin{array}{c}\text { Peso aos } \\
24 \text { meses }\end{array}$ & $\begin{array}{c}\text { Peso aos } \\
30 \text { meses }\end{array}$ \\
\hline Peso ao nascer & - & $0,56(0,56)$ & $0,44(0,49)$ & $0,42(0,42)$ & $0,32(0,43)$ & $0,36(0,43)$ & $0,53(0,76)$ \\
idade adulta
\end{tabular}


pela seleção em comparação às estimativas de herdabilidade e variâncias genéticas.

Nas análises bicaracterísticas e multicaracterísticas, as correlações genéticas entre o peso ao nascimento e os pesos pós-desmame foram menores até os 2 anos de idade e maiores após esta fase. Esse comportamento é pouco esperado, tendo em vista os resultados obtidos por Boligon et al. (2009). Segundo Arango et al. (2002), as magnitudes das correlações entre pesos de vacas dos 2 aos 8 anos de idade diminuíram gradualmente conforme aumentou diferença entre idades. As correlações genéticas estimadas entre pesos obtidos em idades jovens e o peso adulto foram altas. Em gado de corte, de acordo com Mercadante et al. (1995) e Mercadante et al. (2004), as estimativas de correlações genéticas entre pesos em diferentes idades são positivas e de magnitude moderada a alta. Segundo Mascioli et al. (1999), Arango et al. (2002), Talhari et al. (2003) e Mello et al. (2006), as correlações genéticas entre peso adulto da fêmea e peso em idades jovens são positivas e maiores que 0,42 . Portanto, a seleção para maior peso a idades jovens acarretará aumento no peso adulto dos animais.

\section{Conclusões}

Os efeitos maternos influenciam os pesos do nascimento aos 2 anos de idade, e o peso à desmama é o mais afetado pelos efeitos maternos. É fundamental considerar os pesos antes da seleção na obtenção de estimativas de parâmetros genéticos para pesos após a seleção e a análise multicaracterística é a mais indicada. A seleção com base em características de crescimento em qualquer idade deve promover ganhos genéticos moderados no peso corporal de animais da raça Canchim em todas as idades-padrão, inclusive nos pesos ao nascer e à idade adulta das fêmeas.

\section{Referências}

ALBUQUERQUE, L.G.; MEYER, K. Estimates of direct and maternal genetic effects for weights from birth to 600 days of age in Nelore cattle. Journal of Animal Breeding and Genetics, v.118, p.83-92, 2001.

ALENCAR, M.M. Critérios de seleção em bovinos de corte no Brasil. In: SIMPÓSIO NACIONAL DE MELHORAMENTO ANIMAL, 4., 2002, Campo Grande. Anais... Campo Grande: SBMA, 2002. (CD-ROM).

ALENCAR, M.M.; TREMATORE, R.L.; BARBOSA, P.F. et al. Efeitos da linhagem citoplasmática sobre características de crescimento em bovinos da raça Canchim. Revista Brasileira de Zootecnia, v.27, n.2, p.272-276, 1998.

ARANGO, A.; CUNDIFF, L.V.; VAN VLECK, L.D. Genetic parameters for weight, weight adjusted for body condition score, height, and body condition score in beef cows. Journal of Animal Science, v.80, p.3112-3122, 2002.

BALDI, F.; ALENCAR, M.M.; FREITAS, A.R. et al. Genetic parameters for body weight, condition score and body size indices of Canchim cows In: WORLD CONGRESS ON
GENETICS APPLIED TO LIVESTOCK PRODUCTION, 8. 2006, Belo Horizonte. Proceedings... Belo Horizonte: 2006. (CD-ROM).

BARBOSA, P.F. Critérios de seleção para a raça Canchim. In: CONVENÇÃO NACIONAL DA RAÇA CANCHIM, 3., 1997, São Carlos. Anais.... São Carlos: Embrapa-CPPSE; São Paulo, ABCCAN, 1997. p.47-75.

BOLDMAN, K.G.; KRIESE, L.A.; Van VLECK, L.D. et al. A manual for use for MTDFREML. A set of programs to obtain estimates of variances and covariances. Lincoln: Department of Agriculture; Agricultural Research Service, 2002. 129p.

BOLIGON, A.A.; ALBUQUERQUE, L.G.; MERCADANTE, M.E.Z. et al. Herdabilidades e correlações entre pesos do nascimento à idade adulta em rebanhos da raça Nelore. Revista Brasileira de Zootecnia, v.38, n.12, p.2320-2326, 2009.

BULMER, M.G. The effect of selection on genetic variability. American Naturalist v.105, p.201-211, 1971.

CHOY, Y.H.; BRINKS, J.S.; BOURDON, R.M. Repeated-measure animal models to estimate genetic components of mature weight, hip height, and body condition score. Journal of Animal Science, v.80, p.2071-2077, 2002.

CYRILLO, J.N.S.G.; ALENCAR, M.M.; RAZOOK, A.G. et al. Modelagem e estimação de parâmetros genéticos e fenotípicos para pesos do nascimento à seleção (378 dias) de machos Nelore. Revista Brasileira de Zootecnia, v.33, n.6, p.1405-1415, 2004.

DIAS, L.T.; ALBUQUERQUE, L.G.; TONHATI, H. et al. Estimação de parâmetros genéticos para peso em diferentes idades para animais da raça Tabapuã. Revista Brasileira de Zootecnia, v.34, n.6, p.1914-1919, 2005.

DOBSON, A.J. An introduction to generalized linear models Melbourne: Chapman \& Hall, 1990. 174p.

FREITAS, A.R.; FAVORETTI, A.C.; ALENCAR, M.M. et al. Uso da máxima verossimilhança restrita e transformação canônica para estimação de parâmetros genéticos de características de crescimento em bovinos. Revista da Sociedade Brasileira de Zootecnia, v.23, n.3, p.394-401, 1994.

HENDERSON, C.R. Recent developments in variance and covariance estimation. Journal of Animal Science, v.63, p.208-216, 1986.

JOHNSON, Z.B.; BROWN, C.J.; BROWN JR., A.H. Evaluation of growth patterns of beef cows. Fayetteville: Arkansas Agriculture Experimental Station, 1990. (Bulletin, 623).

JOHNSTON, D.J.; CHANDLER, H.; GRASER, H. Genetic parameters for cow weight and condition score in Angus, Hereford, and Poll Hereford cattle. Australian Journal of Agricultural Research, v.47, p.1251-1260, 1996.

KAPS, M.; HERRING, W.O.; LAMBERSON, W.R. Genetic and environmental parameters for mature weight in Angus cattle. Journal of Animal Science, v.77, p.569-574, 1999.

KLOSTERMAN, E.W.; SANFORD, L.G.; PARKER, C.F. Effect of cow size and condition and ration protein content upon maintenance requirements of mature beef cows. Journal of Animal Science, v.27, p.242-251, 1968.

KOOTS, K.R.; GIBSON, J.P.; SMITH, C. et al. Analyses of published genetic parameter estimates for beef production traits. 1. Heritability. Animal Breeding Abstract, v.62, p.309-338, 1994.

LÔBO, R.N.B.; MADALENA, F.E.; VIEIRA, A.R. Average estimates of genetic parameters for beef and dairy cattle in tropical regions. Animal Breeding Abstracts, v.68, p.433-462, 2000.

MASCIOLI, A.S.; TALHARI, F.M.; ALENCAR, M.M. et al. Correlações genéticas entre características reprodutivas e de crescimento de fêmeas da raça Canchim. In: REUNIÃO ANUAL DA SOCIEDADE BRASILEIRA DE ZOOTECNIA, 36., 1999, Porto Alegre. Anais... Porto Alegre, 1999. p.156.

MELLO, S.P.; ALENCAR, M.M.; SILVA, L.O.C. et al. Estimativas de (co)variâncias e tendências genéticas para pesos em um rebanho Canchim. Revista Brasileira de Zootecnia, v.31, n.4, p.1707-1714, 2002 
MELLO, S.P.; ALENCAR, M.M.; TORAL, F.L.B. et al. Estimativas de parâmetros genéticos para características de crescimento e produtividade em vacas da raça Canchim, utilizando-se inferência bayesiana. Revista Brasileira de Zootecnia, v.35, n.1, p.92-97, 2006.

MERCADANTE, M.E.Z.; LOBO, R.B.; REYES, A. Parâmetros genéticos para características de crescimento em zebuínos de carne. Archivos Latinoamericanos de Producción Animal, v.3, p.45-89, 1995.

MERCADANTE, M.E.Z.; RAZOOK, A.G.; TROVO, J.B.F. et al. Parâmetros genéticos do peso no início da estação de monta, considerando indicativo do peso adulto de matrizes Nelore. Revista Brasileira de Zootecnia, v.33, n.5, p.1135- 1144, 2004.

MEYER, K. Estimates of genetic parameters for weaning weight of beef cattle fitting a regression on maternal phenotype. Livestock Production Science, v.52, p.187-199, 1997.

MEYER, K. Estimates of genetic parameters for mature weight of Australian beef cows and its relationship to early growth and skeletal measures. Livestock Production Science, v.44, p.125-137, 1995.

MEYER, K.; CARRICK, M.J.; DONNELLY, B.J.P. Genetic parameters for growth traits of Australian beef cattle from a multibreed selection experiment. Journal of Animal Science, v.71, p.2614-2622, 1993.

MEYER, K.; THOMPSON, R. Bias in variance and covariance components estimators due to selection in a correlated trait. Zeitschrift für Tierzüchtung und Züchtungsbiologie, v.101, p.3350, 1984.

MiszTAL, I. [2001]. REMLF90 Manual. Disponível em: <http:// nce.ads.uga.edu/ ignacy/newprograms.html/>. Acesso em: 6/4/2007.

MONTAÑO-BERMUDEZ, M.; NIELSEN, M.K.; DEUTSCHER, G.H. Energy requirements for maintenance of crossbred beef cattle with different genetic potential for milk. Journal of Animal Science, v.68, n.8, p.2279-2288, 1990.

MUCARI, T.B.; OLIVEIRA, J.A. Análise genético-quantitativa de pesos aos 8, 12, 18 e 24 meses de idade em um rebanho da raça Guzerá. Revista Brasileira de Zootecnia, v.32, n.6, p.1604-1613, 2003.

NEPHAWE, K.A. Application of random regression models to the genetic evaluation of cow weight in Bonsmara cattle of South Africa. South African Journal of Animal Science, v.34, n.3, p.166-173, 2004.

OWENS, F.N.; DUBESKI, P.; HANSON, C.F. Factors that alter the growth and development of ruminant. Journal of Animal Science, v.71, p.3138-3150, 1993.

ROSA, A.N.; LÔBO, R.B.; OLIVEIRA, H.N. et al. Variabilidade genética do peso adulto de matrizes em um rebanho Nelore do estado de São Paulo. Revista Brasileira de Zootecnia, v.29, n.6, p.1706-1711, 2000.

ROSA, A.N.; LÔBO, R.B.; OLIVEIRA, H.N. et al. Peso adulto de matrizes em rebanhos de seleção da raça Nelore no Brasil. Revista Brasileira de Zootecnia, v.30, n.3, p.1027-1033, 2001.

RUMPH, J.M.; KOCH, R.M.; GREGORY, K.E. et al. Comparison of models for estimation of genetic parameters for mature weight of Hereford cattle Journal of Animal Science, v.80, p.583-590, 2002.

SILVA, A.M.; ALENCAR, M.M.; FREITAS, A.R. et al. Herdabilidade e correlações genéticas para peso e perímetro escrotal de machos e características reprodutivas e de crescimento de fêmeas, na raça Canchim. Revista Brasileira de Zootecnia, v.29, n.6, p.2223-2230, 2000 (supl. 2).

TALHARI, F.M.; ALENCAR, M.M.; MASCIOLI, A.S. Correlações genéticas entre características produtivas das fêmeas em um rebanho da Raça Canchim. Revista Brasileira de Zootecnia, v.32, n.4, p.880-886, 2003. 